\title{
DWARF NOVA-LIKE OUTBURST OF SHORT-PERIOD INTERMEDIATE POLAR HT CAMELOPARDALIS
}

\author{
R. Ishioka ${ }^{1}$ and VSNET Collaboration Team
}

Our time-series photometric observations of a short outburst of HT Cam in 2001 strongly suggest that disk instabilities occurred during the outburst.

HT Cam is a cataclysmic variable identified as the optical counterpart of the hard X-ray source RX J0757.0+6306, discovered during the ROSAT AllSky Survey. Tovmassian et al. (1998) suggested that this object is an intermediate polar with a shortest orbital period of $80.92 \mathrm{~min}$ and a spin period of $8.52 \mathrm{~min}$. However, the existence of dwarf nova-like outbursts and the short orbital period allowed an alternative interpretation that it may be an SU UMatype dwarf nova or WZ Sge-type stars (Tovmassian et al. 1998).

After its discovery, 6 outbursts were reported during 4 years. Considering the density of the observations and the shortness of the outbursts, and seeming regularity, the reccurence time of outbursts is about $150 \mathrm{~d}$. This is quite typical among the dwarf novae.

The 6 th outburst was detected by H. Itoh on 29 December 2001, and we started the first time-series observation $0.5 \mathrm{~d}$ after the outburst detection and succeeded in observing during the declining phase of the short outburst. Figure 1 shows the outburst light curve. The rising phase was missed, but the last quiescent observation reported to VSNET was performed within $1.5 \mathrm{~d}$ before the detection. Thus, the rising rate is faster than 3 mag $\mathrm{d}^{-1}$.

In the light curve of the declining phase of the outburst, we detected long-term modulations with a period of $86 \mathrm{~min}$ and strong pulses with a period of $8.6 \mathrm{~min}$. Strong $8.6 \mathrm{~min}$ coherent pulses are white dwarf spin pulses and we identified the $86 \mathrm{~min}$ period as the orbital period. By detecting the spin period, we confirmed the IP classification of HT Cam. The rather short spin period indicates that the magnetic field of HT Cam is not very strong.

Figure 2 shows the average spin profiles of the data on December 30, after subtracting the longer time-scale modulations. At first the amplitude of the spin pulse was $\sim 0.2 \mathrm{mag}$ (a), but it decreased

\footnotetext{
${ }^{1}$ Department of Astronomy, Graduate School of Science, Kyoto University, Sakyo-ku, Kyoto 606-8502, Japan (ishioka@ kusastro.kyoto- u.ac.jp).
}

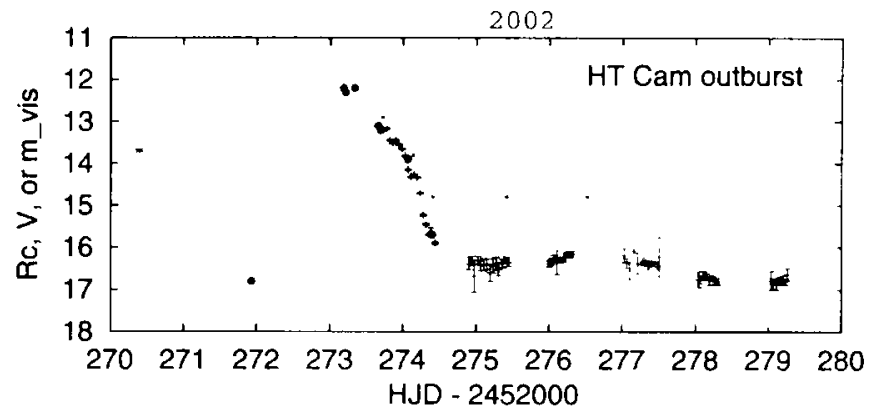

Fig. 1. The light curve of the outburst in 2001. The crosses with, error bars show average magnitude in 1-hr bins of the time-series CCD observations. The filled circles and small crosses show positive detections by visual or snapshot CCD observations and the upper-limits of visual observations reported to VSNET.
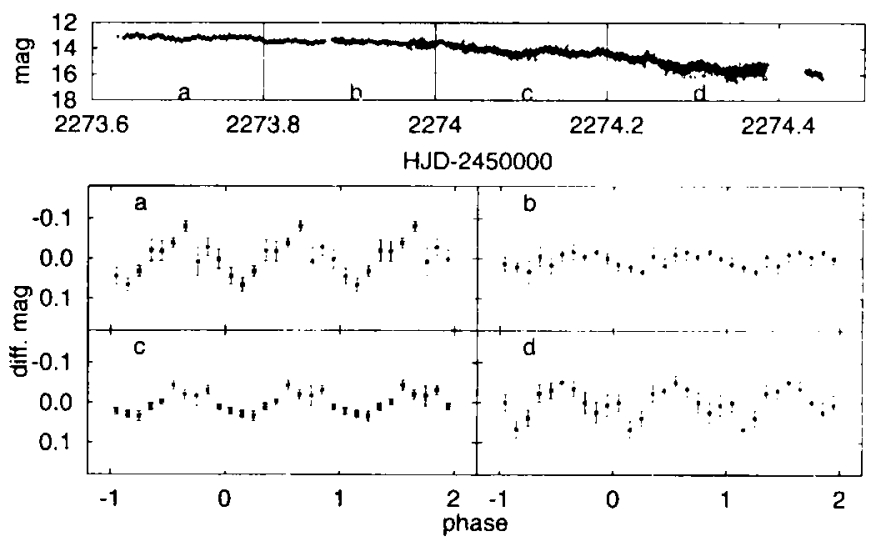

Fig. 2. Averaged spin profiles during the declining phase. The declining rate changed at HJD 2452274.0.

to $\sim 0.1 \mathrm{mag}$ (b). It then gradually increased again during the rapidly declining phase $(\mathrm{c}, \mathrm{d})$.

The discrepancy between the declining rates of the total flux and the pulse flux strongly suggests that disk instabilities occurred during the outburst. Additionally, the regularity of the outbursts suggests that the outbursts on HT Cam are caused by disk instabilities starting at the outer disk edge.

\section{REFERENCES}

Ishioka, R., Kato, T., Uemura, M., Billings, G. II.. Morikawa, K., et al. 2002, PASJ, 54, 581

Tovmassian, G. H., Greiner, J., Kroll, P., Szkudy, P., Mason, P. A., et al. 1998, A\&A, 335, 227 